Relations industrielles

Industrial Relations

\title{
Bouzas Ortiz, J. A., sous la direction de, Libertad sindical
}

\section{Pierre Verge}

Volume 55, numéro 1, 2000

URI : https://id.erudit.org/iderudit/051301ar

DOI : https://doi.org/10.7202/051301ar

Aller au sommaire du numéro

\section{Éditeur(s)}

Département des relations industrielles de l'Université Laval

\section{ISSN}

0034-379X (imprimé)

1703-8138 (numérique)

Découvrir la revue

Citer ce compte rendu

Verge, P. (2000). Compte rendu de [Bouzas Ortiz, J. A., sous la direction de, Libertad sindical]. Relations industrielles / Industrial Relations, 55(1), 183-184. https://doi.org/10.7202/051301ar

Tous droits réservés (C) Département des relations industrielles de l'Université Laval, 2000
Ce document est protégé par la loi sur le droit d'auteur. L’utilisation des services d'Érudit (y compris la reproduction) est assujettie à sa politique d'utilisation que vous pouvez consulter en ligne.

https://apropos.erudit.org/fr/usagers/politique-dutilisation/ 


\section{Libertad sindical}

sous la direction de J. A. BOUZAS ORTIZ, Mexíco: Instituto de investigaciones económicas, Universidad Nacional Autónoma de Mexíco, 1999, 260 p., ISBN 970-92328-1-9.

Intitulé ainsi simplement Libertad sindical, cet ouvrage collectif ne se veut aucunement une présentation systématique, théorique et universelle de cette liberté syndicale; il s'agit plutôt de la juxtaposition d'une douzaine de contributions particulières d'auteurs mexicains soucieux d'exposer les carences de leur droit national en la matière et d'avancer différents correctifs en ces années où il est notamment question de la réforme de la loi fédérale du travail en ce pays.

Les auteurs dont il s'agit sont pour une bonne part des universitaires, juristes, sociologues ou politicologues ; une majorité d'entre eux se rattachent à différents secteurs de la Universidad Nacional Autónoma de Mexíco; on compte aussi quelques avocats syndicaux et un secrétaire du directeur général du travail du District fédéral. S'ajoutent à ce corps essentiellement mexicain, une présentation d'ensemble, relativement systématique, du droit syndical d'Espagne, de madame la professeure Agut Garcia, de même qu'un bref propos de monsieur Tim Beaty, représentant au Mexique du Centre américain pour la solidarité syndicale internationale de la centrale américaine AFL-CIO. Ce dernier se contente d'évoquer de telles difficultés relatives au droit mexicain et de souhaiter plutôt vaguement une collaboration syndicale accrue entre les groupements mexicains et ceux de son pays. Le tout est précédé d'une préface du professeur Néstor de Buen. L'éminent spécialiste du droit du travail mexicain évoque la crise actuelle d'un syndicalisme traditionnellement unitaire et corporatiste face à la présence accrue de regroupements syndicaux totalement (FAT, CSPM), ou partiellement (UNT), en rupture avec le Congrès mexicain du travail (CTM) et face à l' affermissement des politiques néo-libérales de l'État mexicain, de manière à situer ce qui lui paraît constituer en somme une « œuvre de dénonciation $»$.

Le premier texte en cause, qui se voulait, d'après le titre, plus général et théorique (J. A. Bouzas Ortiz et R. M. Mondragon), rappelle succinctement les exigences de la liberté syndicale : pleine autonomie des groupements syndicaux face à l'État et à l'employeur; authenticité de leur représentativité et caractère démocratique de leur vie interne. Non sans avoir évoqué les pressions accrues résultant de la mondialisation et plus particulièrement de l'ALENA, les auteurs en viennent à discuter de difficultés particulières du droit syndical mexicain, comme vont le faire à sa suite, mais d'une façon plus spécifique, les autres collaborateurs mexicains. Compte tenu de la réitération accentuée de ces difficultés dans les différents textes, il convient de tenter d'en faire état immédiatement à partir de l'ensemble de ces contributions. Malgré leur caractère technique, leur existence porte atteinte à l'exercice même de la liberté syndicale :

- L'enregistrement des syndicats, acte qui de simplement déclaratoire et administratif qu'il devrait être, en est venu dans la pratique à constituer un mode de contrôle de l'accès des syndicats à la vie juridique, les organes d'enregistrement se comportant le plus souvent capricieusement en la matière selon les auteurs ; cette procédure devient en quelque sorte alors un mode de contrôle du syndicalisme ;

- Le caractère occulte des conventions collectives : conclues le plus souvent hors la connaissance des salariés, elles se réduisent pour autant, énoncet-on, à des ententes « de protection ", opposables à tout autre syndicat 
prétendant à une représentation authentique du même groupe de salariés ; elles conduisent également au congédiement de syndicalistes dissidents par le biais des clauses d'atelier syndical qu'elles comportent ;

- L'absence de normes destinées à assurer le fonctionnement démocratique des groupements syndicaux, à $y$ contrer en particulier l'appropriation indue de pouvoirs et de fonds par un ou quelques dirigeants, parfois de mèche avec l'employeur ;

- Les faiblesses de la procédure d'établissement de la représentativité syndicale conduisant à la désignation du titulaire du droit de négocier collectivement dans les cas de conflits intersyndicaux de représentation (absence de scrutin secret, pratiques d'intimidation de l'employeur non sanctionnées, liste des personnes aptes à voter établies par ce dernier, etc.) ;

- Le formalisme juridique dans l'exercice du droit de grève, qui a pour effet de notamment rendre illégales les grèves de solidarité, de même que les mesures de répression physique dans le cours des grèves;

- Le manque d'autonomie des tribunaux du travail face aux autorités gouvernementales. En arrière-plan, il y va d'un phénomène général de « corporatisme» qui cherche à s'adapter au nouveau contexte néolibéral et dont on ne prévoit pas la disparition à brève échéance $(\mathrm{M}$. Muñoz, p. 53).

Ces reproches des auteurs, bien incomplètement évoqués, s'accompagnent, a-t-on dit, de suggestions de correctifs précis. Dans son ensemble, comme l'énonce madame la professeure Bensusán, il s'agit d' «identifier un ensemble de réformes institutionnelles indispensables pour accroître la transparence et le caractère démocratique du syndicalisme mexicain [...]» (p. 91).

Certains éléments positifs sont déjà toutefois présents dans le système, dont cet arrêt de la Cour suprême de 1999 , qui reconnaissait, à l'encontre de la législation, l'existence possible de plus d'un syndicat dans la fonction publique fédérale (p. 181 et 182). Il y a également à tenir compte des propositions de réforme du droit syndical avancées par les deux principaux partis d'opposition, le PAN (1995) et le PRI (1998), qui se rejoignent substantiellement. Les auteurs se montrent toutefois critiques à l'égard des propositions issues du processus du dialogue ouvrier-patronal, établi avec la participation du gouvernement mexicain, en vue d'une « nouvelle culture du travail $\gg$ et dont le texte figure en annexe.

On le voit, ces positions critiques des spécialités mexicaines du travail et du syndicalisme s'adressent d'abord et avant tout à un public mexicain et se veulent « agissantes »; de là, comme on l'avait constaté d'emblée, ce caractère souvent technique des textes et aussi cette absence de prétention à l'exposé doctrinal. Le lecteur canadien peut néanmoins y trouver son compte : il sera plus attentif à la réalité de la mise en œuvre du droit du travail mexicain et aux différents courants d'opinion qui ont cours à son sujet. L'ALENA enlève tout exotisme à cette matière... Il demeure toutefois étrange qu' aucun des textes de cet ouvrage collectif portant sur les vicissitudes du droit syndical mexicain ne fasse allusion aux interventions des BAN américain (par exemple, les espèces Sony, US 9403, ou encore Echlin, US 9703) ou canadian (l'affaire Echlin, Can. 98-1), qui, précisément, s'en prennent de façon circonstanciée aux lacunes mêmes que dénoncent les auteurs. L'Accord nord-américain de coopération dans le domaine du travail (ANACT) vaut-il plus que ce silence inquiétant de la part des spécialistes mexicains de la liberté syndicale?

PIERRE VERGE

Faculté de droit Université Laval 